CLAWAR 2018: 21st International Conference on Climbing and Walking Robots and the Support Technologies for Mobile Machines, Panama City, Panama, 10-12 September 2018

\title{
PROPOSAL OF A REMOTE MONITORING SYSTEM OF VITAL SIGNS FOR HEART FAILURE PATIENTS
}

\author{
HECTOR MONTES ${ }^{1,2}$, ELIA CANO ${ }^{3}$, CARLOS ROVETTO $^{3}$, JUAN SALDAÑA ${ }^{3}$, \\ SALVADOR VARGAS ${ }^{1}$ \\ ${ }^{I}$ Faculty of Electrical Engineering, Universidad Tecnológica de Panamá, Panama; \\ ${ }^{2}$ Field and Service Robotics Group, Centre for Automation and Robotics (CSIC-UPM); \\ ${ }^{3}$ Faculty of Systems Engineering and Computer Science, Universidad Tecnológica de \\ Panamá, Panama, hector.montes1@utp.ac.pa
}

\begin{abstract}
The heart failure patients care is increasing, in order to provide the specialized assistance at the right time. Therefore, some researchers have been developed some systems regarding the remote monitoring of the signals of these patients. In this work, it is described a proposal of a remote monitoring system of vital signs, acquired by means of non-invasive biosensors, for heart failure patients. The objective is for monitoring in real-time the vital signs of these patients in order to the specialist doctor to receive the processed data with other added signals and alarms for facilitating his/her diagnosis. This proposal will be developed in Panama, since there is not this kind of system in this country. It will be considered the intrinsic characteristics of the region for this development and also it will be considered the implementation of robotics unit in order to carry out some tasks as patient surveillance, mobile healthcare assistant in rural/remote areas, interface unit between communication systems, etc. However, after to develop this project, it would be extended to other regions of Latin America, since it is very necessary according to statistical data provided by the WHO.
\end{abstract}

Keywords: Heart failure patients; remote monitoring system; vital signs; bio-sensors; wireless data acquisition system.

\section{Introduction}

Cardiovascular diseases (CVD) are the leading cause of death in the world, which caused 15 million deaths in 2015 and have been the leading causes of death during the last 15 years, according to the World Health Organization (WHO) [1]. It is also where the most resources are invested from the public and private sectors worldwide, which in Latin America exceeded 30 billion dollars (in 2015), according to the available information from the WHO offered at the Congress of World Cardiology and Cardiovascular Health (WCC 2016) in Mexico [2].

In the statistical results presented by the WHO in WCC 2016 were indicated some Latin American countries have high rates of death from CVD, including Panama. It was pointed out that heart diseases in Panama affect $29 \%$ of the 
population at a cost of $\$ 300$ million dollars per year [2], which is a very high fee for this country. This is alarming. The Panamanian state must carry out policies for health care in a more forceful way since the percentage destined to deal with this type of diseases is very low, $2 \%$ of the annual budget according to the Social Security Department of Panama (CSS, Spanish language acronym) [3].

Some research works have been developed regarding the remote monitoring systems of patients' vital signs in the last decade, generating positive results. However, these works have been carried out in Europe, USA, Japan, etc., but in Latin America has no reference to any important work in this field. Nevertheless, according to [4] the implementation of patient remote monitoring is an issue that must be further developed in the USA and Europe. Therefore, it is obvious this kind of work must be developed in developing countries, such as Panama.

There are interesting projects regarding to telemedicine, e.g., the Health 2.0 project of the Recover Foundation, which is a non-synchronized telemedicine project through clinical platforms and scientific social networks that is a very effective tool for international cooperation projects in the health development. However, remote monitoring in real time did not used to verify patient variables [5]. On the other hand, there is other system named WANDA (Weight and Activity with Blood Pressure Monitoring System) that uses smartphones to collect data of remote way of heart failure patients [6]. Other related system uses the combination microcontroller-GSM-GPS to collect vital signs of these patients at their homes and send them to the hospital database [7]. Other researchers propose develop cost-effective, easy-to-use remote monitoring systems (using modern technology) for the sake of elderly healthcare and well-being [8], which it can be extended to other patients or people. In addition, currently, it is realized studies to understand challenges and design considerations for a personalized mHealth system that could effectively support heart failure patients after they transition into the home environment. To this end, experiences from users, nurses and physicians are collected [9]. This strengthens our proposal related with remote monitoring system of vital signs for heart failure patients.

In this paper, it is described the proposal of a remote monitoring system of vital signs for heart failure patients that comprises several non-invasive biosensors, data acquisition system, wireless connection, data processing, computers, and higher level addressing-communication capabilities. The rest of the article is organised as follow: in Section 2 a summary of patients' care with heart failure in Panama, making use of the technology is described. Afterward, Section 3 presents the proposal of the remote monitoring system. Finally, conclusions and future works are given in Section 4. 


\section{Overview of patients care with heart failure in Panama using the technology}

There are several systems developed and in developing related to the patients care with heart failure in the world, using the telemedicine concepts, remote monitoring systems, etc., in order to improve the quality of life of them. This section focuses on Panama since the proposed project, presented in this work, will be carried out in this country. However, the authors consider the results of this research will have an international scope.

In Panama, according to a study presented by the WHO in [2], it is the fourth with the number of people with some type of CVD in Latin America with 29\%, behind Chile, Venezuela, and Brazil. There is a wide difference between the number of people with CVD presented by the CSS with the presented by the WHO, which are 80 thousand people [3] and about one million people [2] with CVD, respectively. Therefore, in this project, these numbers will be reviewed, so that a map or a mathematical model of the affected population can be made.

The CSS has launched the Cardiac Failure Clinic (CFC) in 2017 with the aim of reducing mortality due to this type of condition, reducing hospitalizations, optimizing treatment, improving adherence to treatment, educating the patient and reducing the cost of health [3]. The CFC uses the face-to-face attention to the patients and by means of the videoconference. However, most of the patients cannot receive the specialised attention with only one clinic and with this protocol.

Telemedicine programs have been created in some hospital centres, which contain a communication network at voice and data levels, for the care of patients with specialists and general practitioners [10-12]. In the last 10 years, some teleradiology works have been carried out and, occasionally, some electrocardiograms have been sent out, in order to be observed and evaluated by the doctor [11]. This is done from a remote hospital centre to another specialized hospital centre, where both have this program. This makes the speed of attention or monitoring of patients is very low, since these works are clearly face-to-face and the evaluation is deferred.

Telemedicine is the application of ICT to the area of health, wherein this project will be developed, which is the remote monitoring of vital signs of people with CVD risks. However, according to [4] the implementation of remote patient monitoring is an issue that must be further developed in the US and Europe. Therefore, it is obvious to know this kind of project must be carried out in developing countries; such is the case of Panama. 


\section{Proposal of the REMIT system}

REMIT is the acronym of the remote monitoring system of vital signs for heart failure patients. This system consists of non-invasive biosensors, data acquisition system, wireless connection, data processing, computers, and higher-level addressing-communication capabilities. Figure 1 shows the architecture conceptualization architecture of this system.

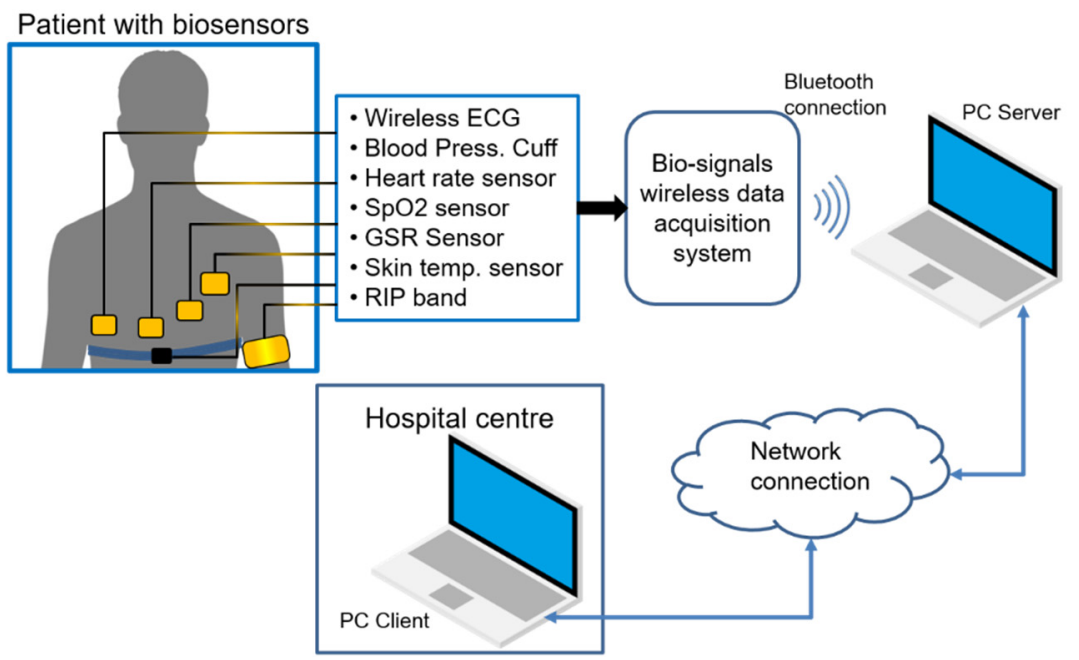

Figure 1. Conceptual Architecture of the REMIT system.

Some non-invasive biosensors are considered for the REMIT system, such as wireless ECG sensor, blood pressure sensor and heart rate sensor as main sensors of the system, but also other sensors can be used such as, pulse oximeter finger clip sensor (SpO2), GSR sensor, respiratory inductance plethysmography (RIP) band, others. Acquiring and processing the signals of these sensors can be obtained from several combinations of human physiological signals. In this case, for monitoring vital signs for heart failure patients.

ECG data and other sensor signals can be seen in real-time on PC server. This collected data is transmitted to this PC through the Bluetooth connection. Therefore, a data analysis after its processing can be carried out, in order to send to the PC client other important data or results together with the real data. After that, at hospital centre, the medical specialist will realize the diagnosis of the patient. 
On the other hand, regarding the monitoring of the vital signs of the patient, in real-time, the doctor will be able an earlier diagnosis of heart failure in him/her. Because some alarms can appear depending on the measure threshold of the biosignals established for each patient, previously settled in the database. With this concept, sudden cardiac arrests can be reduced, since the patient care protocol is activated. It is clear that this methodology must be designed meticulously.

The concepts described above are based when the patient in his/her home. However, the patient can be in any part, e.g., on the street, driving the car, a rural/remote area, etc. In this case, the bio-sensors and the data acquisition system must be connected to other devices, e.g., a smartphone, which replace the PC server in Figure 1. In addition, it is possible to consider an extreme case, where the bio-sensors wireless acquisition system must be connected to another communication mobile system, which can be versatile as a robotics unit for healthcare assistance. Thus, the network connection between the server-client will be Wi-Fi or 3G/4G mobile network. Besides, suppose several patients are being monitored at the same time with different and variable sampling times, depending on the variability their vital signs. Therefore, a server must be connected between the devices worn by the patients (motes) and the PC client, which must be controlled in order to carry out a successful traffic of data. For this, it is essential to have a mathematical model of the patients' distribution, including the main characteristics of their heart diseases, with the aim to have the better decision in the control system. Figure 2 shows the conceptual architecture of the several patients monitored at the same time located in any place, and by different communication systems, depending on their position.

The conceptual architecture summarized in Figure 2 is a complex technical and logistical infrastructure for the patients monitoring with heart failure (obviously, among other kinds of diseases). Therefore, a mathematical model according to the variability of the system must be developed. In this work, the mathematical model proposed will be designed using the Coloured Petri Nets (CPN) [13], since the CPN consist of a graphical representation of an explicit description of states and actions. The model carried out using CPN is useful to work with traffic problems in data networks, since it is necessary to consider the number of data packets transferred and received, the possible loss and overtaking of them, the acknowledgment data, the retransmissions [14], etc.

Initially, a simple CPN model will be designed for the proposed REMIT system presented in this paper, where will be omitted certain parts of the system or will make simplifying assumptions, as shown in the conceptual architecture in Figure 1. Afterward, the CPN model will be gradually modified and extended, 
adding the other omitted parts of the system and updating the first assumptions realized.

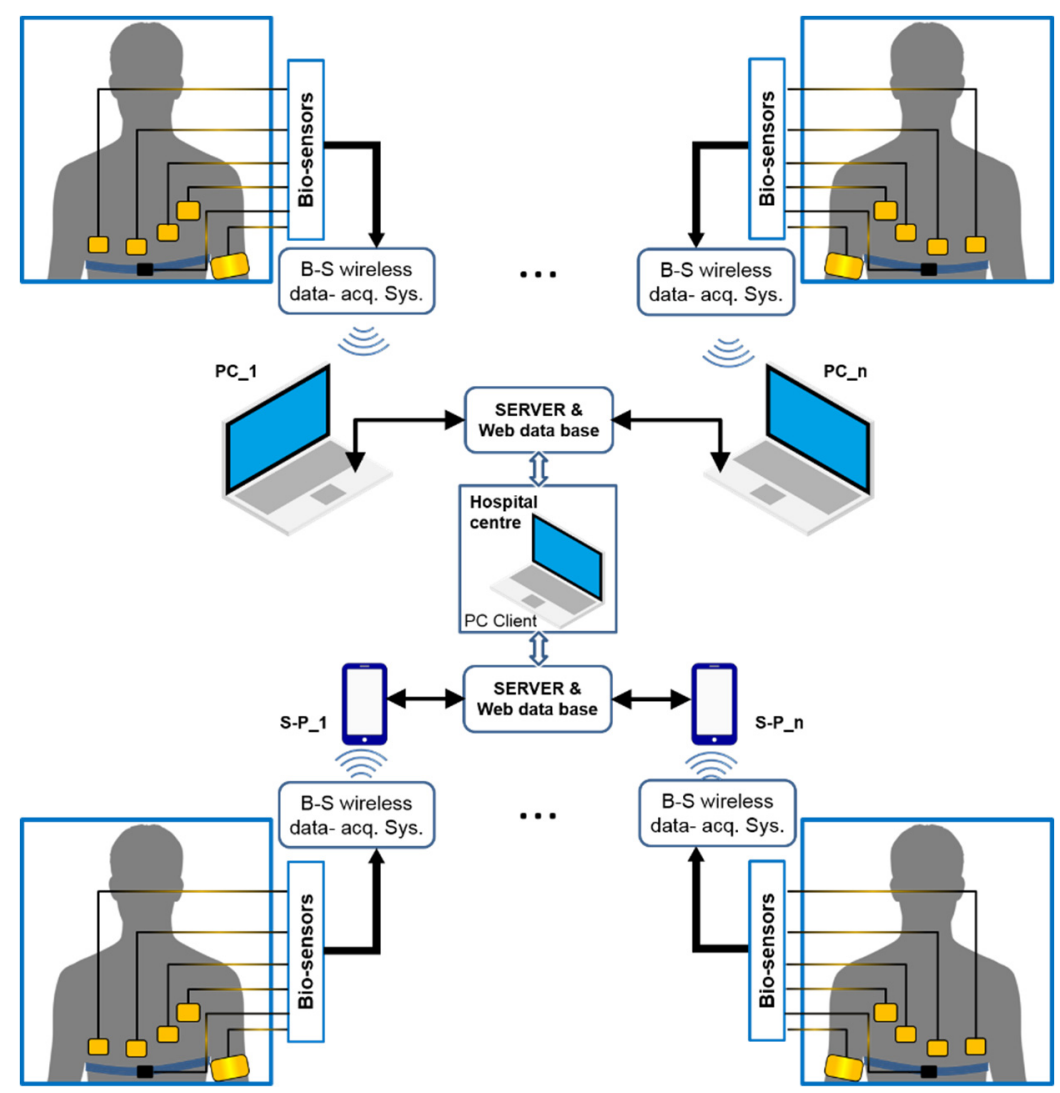

Figure 2. Conceptual Architecture of the REMIT system with several monitored patients.

In Figure 2 the robotics unit can be added in order to detect bio-sensory data through the wireless connection, and other signals of the environment where the patients are. First, the robotics unit can be considered to be installed in rural/remote areas as a mobile healthcare assistant and, in second, as patient surveillance assistant in some hospital that requires data from the patients in real time. Some studies related with the abovementioned can be revised in $[15,16]$. However, in Panama case, a rigorous study must be carried out in order to adapt efficiently all mentioned concepts in this paper, for the patient health care and other related issues. 


\section{Conclusions}

In this paper has been described a proposal of a remote monitoring system of vital signs for heart failure patients with the objective to be implemented in Panama. However, this proposal can be extended outside this country border, where this technology not has been developed nowadays.

With the described in this paper, it is evident the need to develop this kind of system in Panama and other countries of Latin America. With the implementation of the REMIT system, the probability of reduction of cardiac arrests will be possible. However, the patient care protocol must be designed and established painstakingly.

The work that will be carried out is extensive and will be divided into several stages since the first experimental tests with the biosensors and the wireless data acquisition system up to the first connection of two or more patients (can be healthy individuals) within a controlled environment. Afterward, the new stages will be added, with more patients in different positions in several places of the city or the country.

\section{Acknowledgements}

The authors thank the support of the Universidad Tecnológica de Panamá for the development of this project. Hector Montes, Elia Cano, Carlos Rovetto and Salvador Vargas thank the National System of Researchers (SNI-SENACYT) of Panama by the support to their members. Hector Montes also acknowledges support from Centre and Automation and Robotics CSIC-UPM.

\section{References}

1. WHO, The top 10 causes of death. Media centre, WHO. (January 2017.) Available in: http://www.who.int/mediacentre/factsheets/fs310/es/

2. World Heart Federation, El costo de las enfermedades cardiacas en América Latina. Congreso Mundial de Cardiología y Salud Cardiovascular (WCC 2016), México (2016).

3. CSS, Clínica de falla cardiaca inicia atención en el complejo metropolitano. Nota de Prensa de la CSS, (February 2017). Available in: http://www.css.gob.pa/web/8-febrero-2017car.html

4. R. Alvez, Aplicación de Telemedicina para la mejora de los sistemas de emergencias y diagnósticos clínicos. Memoria de trabajos de difusión científica y técnica 9,91 (2011).

5. D. Pérez-Machón, Telemedicina, una red social médica de ayuda humanitaria entre España y Camerún. Gaceta Sanitaria 29(1), 59 (2015). 
6. M. Lan, L. Samy, N. Alshurafa, M-K Suh, H. Ghasemzadeh, A. Macabasco, M. Sarrafzadeh, WANDA: An End-to-End Remote Health Monitoring and Analytics System for Heart Failure Patients. Proc. of the Conf. on Wireless Health WH'12, 9:1 (2012)

7. M. Arunkumar, Remote Monitoring of Vital Signs in Chronic Heart Failure Patients. Intl. J. of Innovative Research in Computer and Comm. Eng. 2(1) (2014).

8. S. Majumder, T. Mondal, and M. J. Deen, Wearable Sensors for Remote Health Monitoring. Sensors 2017 17, 130 (2017).

9. N. Alnosayan, S. Chatterjee, A. Alluhaidan, E. Lee, L. Houston, Design and Usability of a Heart Failure mHealth System:A Pilot Study. JMIR Human Factors 4(1), e9 (2017).

10. CSS, Implementación del proyecto de telemedicina en Policlínica de Santiago. Nota de Prensa de la CSS. (April 2016) Available in: http://www.css.gob.pa/web/5-abril-2016santiago.html

11. F. Arias, Pasos que ha dado Panamá en el tema de telemedicina. Avance Tecnológico en Salud, La Prensa. (June 2016) Available in: https://www.prensa.com/salud_y_ciencia/Pasos-dado-Panamatelemedicina_0_4513048767.html

12. Estrella de Panamá, CSS refuerza la telemedicina. Noticias, La Estrella de Panamá. (December 2016). Available in: http:/laestrella.com.pa/ panama/nacional/refuerza-telemedicina/23977136

13. K. Jensen, Coloured Petri nets. IEE Colloquium on Discrete Event Systems: A New Challenge for Intelligent Control Systems, London, 1993, pp. 5/1-5/3.

14. K. Jensen and L.M. Kristensen, Coulored Petri Nets, Modelling and Validation of Concurrent System, (2009)

15. K. Boman, M. Olofsson, P. Berggren, P. Sengupta and J. Narula, RobotAssisted Remote Echocardiographic Examination and Teleconsultation. JACC: Cardiovascular Imaging 7(8), (2014).

16. E. Wicklund, Telemedicine robots: Out of science fiction and into the mainstream. mHealth Intelligence. (October 2017) Available in: https://mhealthintelligence.com/features/can-telemedicine-robots-movefrom-fantasy-to-fact 